\title{
Optimization techniques for Transportation Problems
}

\author{
Gauthaman. $\mathrm{P}^{1}$ \\ ${ }^{1}$ Assistant Professor, Prist University, Puducherry
}

\begin{abstract}
This paper infers about optimization technique for various problems in transportation engineering. While for pavement engineering, maintenance is priority issue, for traffic it is signalling which is priority issue. Many optimization methods are discussed though given importance of genetic algorithm approach. While optimization techniques nearly approach practicality, research works are on for modern optimization techniques which not only adds ease of structure but also provide compatibility to modern day problems encountered in transportation engineering. Some of the modern tools are discussed to employ optimization techniques which are quite simple to use and implement once it is calibrated to the desired objective.
\end{abstract}

\section{INTRODUCTION}

Transportation engineering faces challenges in the field of decision making for current transportation problems which is briefly discussed. Optimization techniques could be employed for such problems and one can ensure definite solutions to such problems. Genetic algorithm can be used effectively in transportation engineering for solving such problems with stipulated time.

\section{TRANSPORTATION PROBLEMS AND SOLUTIONS}

Nowadays one has to encounter many transportation problems in hand and as transportation engineer or planner has to search for better solution to the specific problem. Though wide range of solutions can be available, one has to opt for optimum solution which might be easily available at finite point of time. In terms of pavement engineering, optimal solution is needed to get maintenance scheduling for a long stretch of rural roads. In terms of traffic, signalling is to be done to minimize the unnecessary delays at intersections. For transportation planning also, this optimization technique proves to be effective in scheduling.

\section{OPTIMIZATION TECHNIQUES}

Basically two models are encountered in optimization either constrained or unconstrained problem. In most of the cases constrained is applied in real life problems. Unconstrained model could be solved by linear regression model or other techniques. Constrained model should be solved by higher optimization techniques such as Genetic algorithm wherein solution could be got in fixed interval of time. Signalling of traffic lights is primarily important in urban environment where automated signalling system such as SCOOT uses optimized solutions. Traffic signal optimization on road arteries consists of two problems: the solution algorithm and the progression model used to compute the values of the objective function1. The above is one example to solve problems using optimization techniques. Thus it is clear that for modern day use of technologies optimization techniques is vital in deciding the traffic solutions in real time. Use of optimization is not limited to traffic scheduling but for pavement maintenance and scheduling also, this proves effective in decision making process.

\section{GENETIC ALGORITHM IN TRANSPORTATION ENGINEERING}

A bi-level programming model for the bus frequency design is presented, which determines the optimal bus frequencies aiming to minimize the total travel time of passengers subject to the constraint on the overall fleet size of each company by accounting for the route choice behaviours of the users. An iterative approach, which consists of a genetic algorithm and a label-marking method, is used to solve the bi-level model. The results show that the optimization can improve the local service level of one company, and the proper integration of several companies probably improves the efficiency of resources and the service level of the whole transit system2. At the National University of Singapore (NUS), Tan (1995) discussed the applications of a natural evolutionary algorithm for pavement management activities optimization. Initially he used Gas for a highway routine maintenance optimization problem at network level studied by Fwa et al.(1988). He showed that GA can handle the network optimization problem easily and it produced better results. Like most of the engineering optimization problems the example problem was highly constrained. He developed models such as PAVENET and PAVENET-R. 


\section{GENETIC ALGORITHM TOOLBOX}

Genetic algorithm and Direct Search Toolbox extends the optimization capabilities in MATLAB and the Optimization Toolbox with tools for using the genetic and direct search algorithms. We can use these algorithms for problems that are difficult to solve with traditional optimization techniques, including problems that are not well defined or are difficult to model mathematically. We can also use them when computation of the objective function is discontinuous, highly nonlinear, stochastic, or has unreliable or undefined derivatives.
The Genetic Algorithm and Direct Search Toolbox complement other optimization methods to help us find good starting points. Toolbox functions, which can be accessed through a graphical user interface (GUI) or the MATLAB command line, are written in open MATLAB language. This means that we can inspect the algorithms, modify the source code, and create our own custom functions. The GA Toolbox is as shown in figure

1. Thus it makes simple to use the platform once objective function and constraints are set.

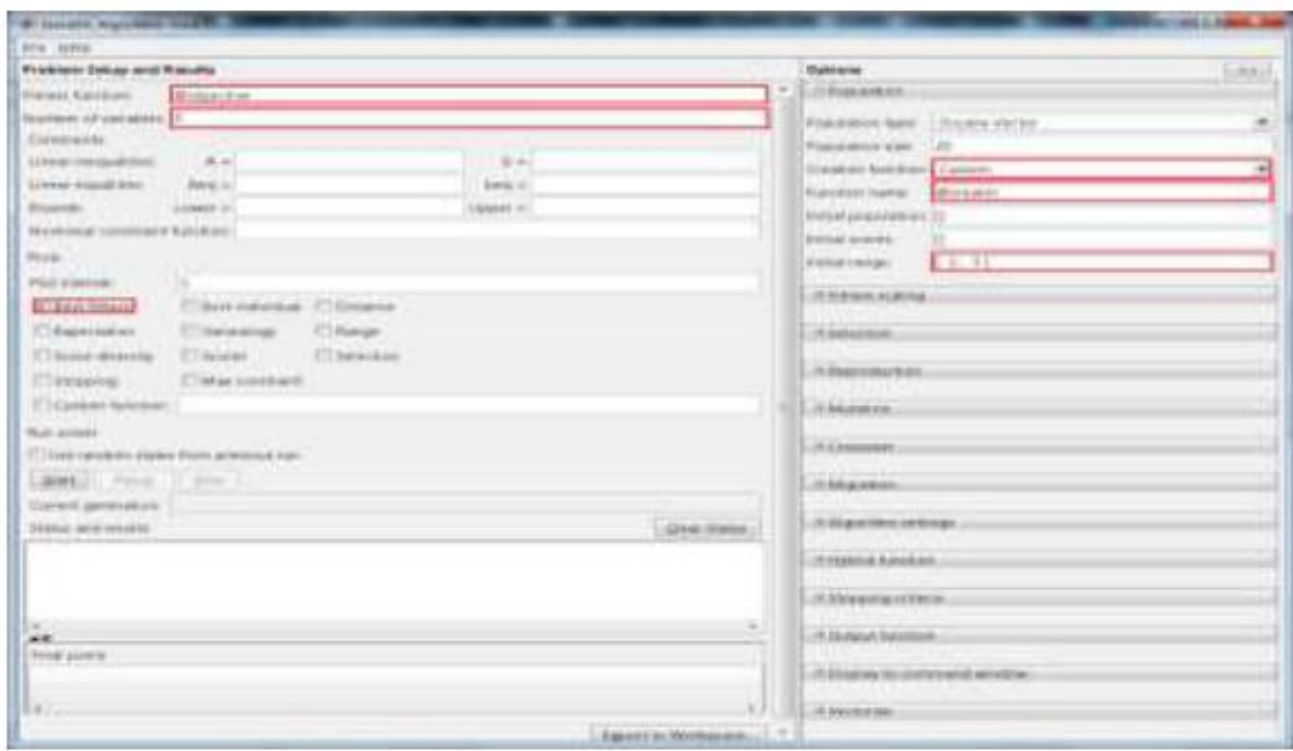

Fig 1. Genetic Algorithm Toolbox

\section{PARETO-OPTIMAL SOLUTIONS}

Till now we have discussed about single objective function with constraints, sometimes it is needed to solve multiple objective functions with multiple constraints. Paretooptimal solutions are what we use to solve multiple objective functions.

\section{CONCLUSIONS}

Therefore Optimization techniques are essential in handling day to day problems and solutions. It is also important to use in a proper manner to avoid misrepresentation of solutions.

\section{REFERENCES}

[1] A global optimization approach to solve the traffic signal synchronization problem,

[2] L.Adacher, Roma Tre University, Roma, Italy, www.sciencedirect.com

[3] Genetic Algorithm for Bus Frequency Optimization, Bin Yu;Zhongzhen Yang; and Jinbao Yao, Journal of Transportation Engineering, ASCE
[4] Optimal Routing and Scheduling in Transportation: Using Genetic Algorithm to Solve Difficult Optimization Problems by Partha Chakraborty 